\title{
Varieties of Indefinites
}

\author{
Donka F. Farkas \\ University of California, Santa Cruz
}

\section{Introduction}

Languages that have determiners often have a rich inventory of them. In English, indefinite determiners include $a(n)$, some, a certain, this, one, another, cardinals, partitives, the zero determiner of bare plurals (in some analyses), and, according to Horn 1999 and Giannakidou 2001, any. Despite the attention indefinites have received in the literature, characterizing what is common to all of them and what is specific to each is still an elusive task. This paper investigates the first three determiners in this list, attempting to provide a semantic characterization that accounts for their distribution. (For pertinent discussion of issues that overlap to some extent with those taken up below, see Kamp and Bende-Farkas 2002, and for a discussion of the French versions of some and a certain, see Jayez and Tovena (this volume).)

The Ds that concern us here share two characteristics: (i) they are indefinite, and (ii) they are existential when not in the scope of any operator or quantifier. As seen in [1], they may occur as pivots in existential there constructions, and therefore all three form weak DPs.

[1] There's a/some/a certain mistake here somewhere.

The indefinite nature of these Ds will be taken for granted in what follows. I stay neutral with respect to whether the definite/indefinite distinction is a matter of novelty/familiarity, uniqueness/non-uniqueness or some third property that subsumes these two.

Our three indefinite Ds contrast in various ways. With respect to scope, for instance, only $a(n)$ may occur within the immediate scope of negation or as a generic, and a certain, but not the others, is subject to restrictions on its modal interpretation. Sections 3 - 5 propose a semantic characterization that accounts for this concentric distribution pattern, as well as for other peculiarities that are brought up in the course of the discussion. These core sections are sandwiched between an introductory and a concluding section.

\section{Framework}

\subsection{Files, contexts and context change}

I follow FCS and DRT in assuming that the expressions to be interpreted are representations $\phi$, which I refer to here as files. (Files are equivalent to DRSs.) Files are constructed on the basis of syntactically analyzed utterances. A simple file consists of a set $X_{\phi}$ of indexed variables (the domain of $\phi$ ), and a set $C_{\phi}$ of conditions. Both components should be further structured even in simple files. The elements of the 
domain of a file should be structured in terms of their saliency or topicality. In what follows, however, differences in topicality are ignored since they do not appear to be relevant to the subset of indefinites we are investigating here. ${ }^{1}$ With respect to conditions in a simple file, I assume a distinction between value conditions and main conditions. The role of the former is to specify a set, called value set, from which values of a variable are to be chosen. Value conditions play the role of restrictive conditions on variables in restricted quantification logics, whose advantages for linguistics were first pointed out in McCawley $1981 .^{2}$ Complex files involve files with auxiliary (subordinate) files as constituents. I follow here DRT (rather than FCS) in assuming that these auxiliary files survive in final structures. Auxiliary files are introduced by quantifiers, operators and complement-taking predicates. ${ }^{3}$

The interpretation of files is done relative to a model $\mathrm{M}=\langle\mathrm{D}, \mathrm{W}, \mathrm{I}, \mathrm{H}, \mathrm{A}\rangle$, where $\mathrm{D}$ is a set of entities, $\mathrm{W}$ is a set of worlds, each with its own domain, $\mathrm{D}_{w}$, a subset of $\mathrm{D}, \mathrm{I}$ is an interpretation function assigning denotations to predicates, $\mathrm{H}$ is a set of assignment functions, and $\mathrm{A}$ is a set of accessiblity relations on worlds. Assignment functions are partial functions from variables and world domains to an element of the domain in question. The notions of truth and satisfaction conditions are defined as in [2] and [3] respectively.

[2] $\phi$ is true in $w$ iff there is an $f \in \mathrm{H}$ such that $f$ satisfies $\phi$ relative to $w$.

[3] $f$ satisfies $\phi$ relative to $w$ iff for every $x_{n} \in X_{\phi}, f\left(x_{n}, D_{w}\right)$ meets the conditions in $\phi$ relative to $w$.

Complexity at the interpretation level involves evaluating auxiliary files by auxiliary functions other than the initial function or at world domains different from that of the initial world. Auxiliary functions extend the initial function on particular variables. The world domains are domains of worlds made accessible to the initial world by accessibility relations in A. We encounter the former type of complexity in discussing quantificational Ds in the next section, and the latter when we discuss the modal restrictions on a certain in subsection 5.2.

Crucial to both DRT and FCS is that the semantic characterization of an expression $e$ involves defining its file change potential. Below I follow Heim 1982, Chapter 3 in taking the common ground of a conversation to be a file $\phi$. The context of $\phi$ is defined as in [4] (cf. (Heim 1982, Groenendijk, Stokhof and Veltman 1996, Abusch and Rooth 1997):

[4] $\mathrm{c}(\phi)=\{\langle w, f\rangle: f$ satisfies $\phi$ in $w\}$

The world - assingment function pairs in the context of a file relative to a model $\mathbf{M}$ are the possibilities of $\phi$ in M.

The Stalnakerean notion of the context set of $\phi$ is defined in [5] to be the set of worlds that figure in the context of $\phi$.

[5] $\mathrm{Wc}(\phi)=\{\mathrm{w}$ : there is an $f \in \mathrm{H}$ such that $\langle w, f\rangle \in \mathrm{c}(\phi)\}$

To see how Stalnaker's notion of context change is captured, assume that an input file $\phi$ is changed into an output file $\phi \prime$ by uttering an expression $e$. The context of the output file, $c(\phi)$, is defined as in [6]: 
[6] $\mathrm{c}(\phi \prime)=\left\{\left\langle w, f^{\prime}\right\rangle\right.$ : there is a possibility $\langle w, f\rangle \in \mathrm{c}(\phi)$ and $f \prime$ is a (possibly trivial) extension of $f$ that satisfies $\phi \prime$ in $\mathrm{w}$ \}

The context set of $\mathrm{c}\left(\phi^{\prime}\right)$ is the set of worlds in the input context that survive into the new one, i.e., those worlds in $\operatorname{Wc}(\phi)$ relative to which the new context $\phi \prime$ is true. The effect of an expression on its context boils down to how the addition of the expression in question to a file $\phi$ affects a possibility $\langle w, f\rangle$ in the set of possibilities of its input context, $\mathrm{c}(\phi)$.

\subsection{A constraint-based approach to Ds}

Here we are interested in full-fledged, that is, unincorporated, argumental, that is, non-predicative, descriptive, that is lexical N-headed, DPs. Minimally, the contribution of such DPs to a file consists in (i) a variable (aka a discourse referent) and (ii) a value condition. I assume that the $\mathrm{D}$ is responsible for the former, and the NP for the latter. The role of the NP is constant across all types of argumental descriptive DPs. We are concerned here with the question of what distinguishes various Ds from one another. In the view advocated here, part of the answer involves the claim that Ds may be lexically marked for particular constraints they impose on the variable they introduce. The distribution of a DP whose D is a determiner $d$ is restricted by these constraints in that the DP may occur only under interpretations that meet them, and in environments that make these interpretations possible. A similar position is taken with respect to NPIs in Giannakidou 1998. It is the standard one when it comes to distinguishing between definite and indefinite Ds, i.e., between $a(n)$ and the in English. According to Kamp 1981 and Heim 1982, the constraint involved is an 'input context constraint' in that it concerns the status of the variable relative to the input file: definite Ds require the variable to be one that is already present in the input file, while indefinite Ds require the variable to be new (or, alternatively, simply lacking the definiteness requirement). Under the uniqueness view of definiteness, definite Ds impose a constraint on the value set, requiring it to be singleton. Another example of a value set constraint is found in Kadmon and Landman 1993, involving the widening of the value set of any. I assume that Ds may in principle impose both input context constraints and value set constraints, but will not discuss either here.

I suggest below that Ds may also impose constraints on the identity and properties of the function (or set of functions) that assigns values to the variable the $D$ introduces. I call this type of constraints functional constraints. We meet functional constraints in the next subsection, when discussing quantificational Ds and dependent indefinites and again in subsection 4.1., where the quantificational force of some indefinites is discussed.

Another type of constraint that plays a role in the discussion below, termed output context constraints, involves the status of the variable introduced by the $\mathrm{D}$ relative to the output context. In Section 4.2. I argue that constraints of this type are part of a complete characterization of some, and in Section 5 it is shown that they are crucial in capturing the distribution and interpretation of a certain. 


\subsection{Quantificational vs. non-quantificational determiners}

I follow DRT and FCS in taking the fundamental distinction between quantificational and non-quantificational Ds to involve the issue of the possible further effect of a D on a file, beyond the introduction of a variable. I follow DRT in assuming that quantificational D, exemplified in [7] by every, trig creation of a tripartite complex file as in [7b].

[7] a. Every man left.

b. $\left[\left[x_{3}: \operatorname{man}\left(x_{3}\right)\right]_{\phi^{\prime}} \forall_{3}\left[\operatorname{leave}\left(x_{3}\right)\right]_{\phi^{\prime}}\right]_{\phi}$

The satisfaction conditions of [7b] are given in [8]:

[8] $f$ satisfies $\phi$ in $w$ iff every extension $f_{3}$ of $f$ on $x_{3}$ that satisfies $\phi \prime$ in $w$ is such that $\phi "$ is true relative to $f_{3}$ in $w$.

By [2], the last part of this requirement amounts to the condition in [9]:

[9] $\phi "$ is true relative to $f_{3}, w$ iff there is a (possibly trivial) extension $g_{3}$ that satisfies $\phi "$ relative to $w$.

The effect of every on the satisfaction conditions of the complex structure it contributes is to introduce a set of auxiliary functions $\mathrm{F}_{n}$ defined with the help of the expression in the Restrictor, $\phi \prime$, and to require the expression in the Nuclear Scope, $\phi "$, to be true relative to each function $f_{n} \in \mathrm{F}_{n}$. In the notation adopted here, the set of functions $\mathrm{F}_{n}$ are functions that extend a function $f$ over the value set of a variable $x_{n}$ by assigning it each of the elements in the value set in turn. A set of functions that makes a variable cover its value set in this way is called the maximal set of extensions of $f$ on $x_{n}$ relative to the value condition on $x_{n}$, defined as in [10]:

[10] $\mathrm{F}_{n}$ is a maximal set of extensions of $f$ on $x_{n}$ relative to a value condition $C$ specifying a value set $P$ iff $\forall f_{n} \in \mathrm{F}_{n}, f_{n}$ extends $f$ relative to $x_{n}$ and $f_{n}$ meets $C$, and for every $s \in P$, there is an $f_{n} \in \mathrm{F}_{n}$ such that $f_{n}=s$, and for every two functions $f_{n}, f \prime_{n} \in \mathrm{F}_{n}, f_{n}\left(x_{n}\right) \neq f \prime_{n}\left(x_{n}\right)$.

As a notational convention, a set of functions that make a variable cover its value set, as well as the functions in such a set, are subscripted for the variable in question.

The evaluation requirement imposed by every on the quantificational structure it introduces is given in [11],

[11] For every $f_{n} \in \mathrm{F}_{n}, \phi "$ is true relative to $f_{n}$ and $w$.

where $\phi "$ is the representation of the expression in the NS, and $\mathrm{F}_{n}$ extends $f$ maximally on $x_{n}$ relative to its value condition, given by the expression in the Restrictor.

The effect of every on the evaluation fate of the variable it introduces is to have it be given values by the functions in $\mathrm{F}_{n}$. Common to all quantificational $\mathrm{Ds}$ is that they impose a tripartite structure of the type in [7b], where the Restrictor is contributed by the NP sister of the D and the Nuclear Scope is contributed by the 
rest of the sentence. The effect on the satisfaction conditions of the resulting file is to introduce a set of functions $\mathrm{F}_{n}$ maximally extending the input function $f$ on $x_{n}$ relative to the value condition of $x_{n}$. As a result, $x_{n}$, the variable introduced by the quantificational $\mathrm{D}$, is given values by $\mathrm{F}_{n}$. The functional constraint quantificational Ds impose on the variable they introduce is that of being assigned values by the functions in $\mathrm{F}_{n}$. I am also assuming that such Ds require the value set of the variable they introduce to be non-singleton in at least one possibility in their output context.

The effect of non-quantificational Ds on the file is simple: they only introduce a variable. The simple structure is connected to the fact that such Ds do not involve the introduction of auxiliary functions. The rest of this paper examines some ways in which non-quantificational Ds differ from one another.

\section{The unmarked singular indefinite $a(n)$}

The Kamp/Heim characterization of indefinites is, I claim, correct for $a(n)$. I rephrase it as in [12], following Hawkins 1991 and references cited therein, according to which the indefinite nature of $a(n)$ is due to the absence of a definiteness requirement, rather than to the presence of an indefiniteness constraint.

[12] The $\mathrm{D} a(n)$ is unmarked in the sense that it contributes no constraints on the variable it introduces. ${ }^{4}$

The unmarked nature of $a(n)$ is key to its versatility. Since there are no constraints on its functional fate, a variable introduced by $a(n)$ is available for unselective binding and may occur within the scope of any operator or predicate. We therefore predict freedom of interpretation and distribution. This freedom is exemplified below looking at three special cases: dependent indefinites, generic indefinites, and indefinites within the immediate scope of negation.

(a) Dependent (co-varying) indefinites

In [13], where DPs are subscripted with the index of the variable introduced by their $\mathrm{D}$,

[13] Every student ${ }_{i}$ read a poem $_{j}$.

the evaluation functions of $x_{i}$ are the functions in $\mathrm{F}_{i}$, introduced by every. The sentence is ambiguous due to the fact that $x_{j}$ is either evaluated by the input function, $f$, resulting in the wide scope reading of the indefinite relative to the universal, or it is evaluated by the functions in a set $\mathrm{G}_{i}$, which are functions that extend those in $\mathrm{F}_{i}$ on $x_{j}$. Under this interpretation, the indefinite has narrow scope relative to the universal. ${ }^{5}$

The functions in $G_{i}$ are the functions relative to which the expression in the $\mathrm{N}$ (uclear) $\mathrm{S}$ (cope) has to be true. Each function in $\mathrm{G}_{i}$ is an extension of a function in $\mathrm{F}_{i}$. The subscript here indicates that the functions in the set make $x_{i}$ cover its value set. Under the narrow scope reading of the indefinite in [13], there is a special relation between $\mathrm{F}_{i}$ and $\mathrm{G}_{i}$, characterized in [14]:

[14] For every $v \in D$ such that there is an $f_{i} \in F_{i}$ and $f_{i}\left(x_{i}\right)=v$, there is a unique function $g_{i}^{v} \in G_{i}$ that extends $f_{i}$ on $x_{j}$. 
In cases such as these the values of $x_{i}$ will be called evaluation parameters for $x_{j}$. Ds that require dependency

As a first example of a marked indefinite, we take a brief look at the reduplicated form of the indefinite article in Hungarian, discussed in detail in Farkas 1997. In this language, besides the unmarked singular indefinite egy, corresponding to $a(n)$, there is also a morphologically marked form, egy-egy, exemplified in [15]:

[15] Minden diák olvasott egy-egy verset.

every student read.Past a-a poem.Acc

'Every student read a poem.'

Unlike [13], [15] is unambiguous: the reduplicated indefinite must be interpreted within the scope of the universal. I suggest that this difference is due to the fact that the reduplicated indefinite determiner is a dependent indefinite in the sense that it imposes on the variable it introduces the functional constraint in [16]:

\section{[16] Dependency constraint}

The variable $x_{j}$ introduced by a dependent $\mathrm{D}$ must be evaluated by functions in a set $G_{i}$, where $i \neq j$.

This constraint can be met only in environments where another expression contributes a set of functions that makes another variable cover its value set. In order to meet the constraint, the variable introduced by the reduplicated $\mathrm{D}$ will have to be within the semantic scope of this expression. We thus correctly predict the lack of ambiguity in [15] as well as the fact that egy-egy indefinites are not felicitous in [17], where there is no variable for egy-egy to depend on.

[17] *Mari meglátogatott tegnap délután egy-egy ismerőst.

Mari visited yesterday afternoon an-an acquaintance

In this approach, dependent indefinites are marked for dependency while unmarked indefinites are compatible with it. The dependency requirement of the reduplicated indefinite in Hungarian limits the distribution of these DPs to environments where the requirement can be met. In such environments, the possible interpretations are limited to those in which the constraint is met.

(b) Generic indefinites

As is well-known, ordinary indefinites in English (as well as in many other languages) have generic readings, exemplified in [18].

[18] A seagull is intelligent.

I assume here that the semantic representation of [18] is as in [19], where the generic adverb GEN unselectively binds the variable $x_{3}$ introduced by $a$ in $a$ seagull.

[19] $\mathrm{GEN}_{3}\left[x_{3}: \operatorname{seagull}\left(x_{3}\right)\right.$ intelligent $\left.\left(x_{3}\right)\right]$

A variable bound by GEN ranges over default values of its value set because GEN has the effect of introducing a set of auxiliary functions that maximally extend the input function on the variables bound by it on the default values of their value sets. 
Given what was said above about dependent indefinites, it is predicted that they cannot occur as generics. That this prediction is correct is shown by the ungrammaticality of [20] under a generic interpretation. ${ }^{6}$

[20] *Egy-egy sirály okos. a-a seagull intelligent

(c) Indefinites within the immediate scope of negation

As shown in [21], unmarked indefinite DPs may occur within the immediate scope of negation.

[21] Mary didn't buy an apartment in San Francisco when she could have afforded it and now it is too late.

I assume that sentence negation gives rise to complex files of the form in [22]:

[22] $\left[\neg \phi^{\prime}\right]_{\phi}$

The satisfaction conditions of such files are as in [23]:

[23] A function $f$ satisfies $\phi$ iff no extension $f_{i, \ldots, n}$ of $f$ on the variables $i, \ldots, n$ in $\mathrm{X}_{\phi^{\prime}}$ satisfies $\phi^{\prime}$.

A variable is 'in the immediate scope of negation' iff its index is on the set of functions introduced by the negative operator. In this case the variable covers its value set. This may happen only if the variable is in the domain of the file the negation is prefixed to. A variable has wide scope relative to negation if it is assigned values by $f$. A variable is within the scope of negation but not within its immediate scope if it occurs within the domain of a file subordinate to the negated one.

Given what was said above, we predict that dependent indefinites may not introduce variables that are within the immediate scope of negation, since such variables do not meet the dependency constraint. That this prediction is correct is shown by the ungrammaticality of [24].

[24] *Mari nem látott egy-egy sirályt.

M. not see.Past a-a seagull.Acc

Mary did not see a seagull.

A common characteristic of generic indefinites, indefinites within the immediate scope of negation, and quantificational DPs is that the variable they introduce covers its value set. In the notation used here this means that the variable is evaluated by a possibly non-singleton set of functions coindexed with it. (The fact that the set of functions is non-singleton correlates with the fact that the value set of the variable is not singleton either.) Unique to quantificational DPs is that the functions that evaluate the variable in question are introduced by the quantificational D.

To sum up so far, the claim made here is that $a(n)$ is the unmarked singular determiner in English, which is what explains its versatility. If this is so, the other Ds have to be marked relative to it and therefore will have a more limited distribution. The discussion of the reduplicated indefinite $D$ in Hungarian is a first example of a 
marked D. In the next two sections we turn to two marked Ds in English, singular some and a certain.

\section{Singular some ${ }^{7}$ \\ 4.1. Scope and quantificational force}

As shown by the scopally ambiguous examples in [25], some indefinites, just like their unmarked sister, may co-vary with an individual or situational variable and may have narrow scope relative to dispositional and intensional predicates, as well as with respect to conditionals, without being required to occur within the scope of any of these expressions.

[25] a. Every Sunday they chose some hymn that was out of their range. (Garrison Keillor's Prairie Home Companion broadcast 04/14/01)

b. Each life converges to some center.

c. In target of opportunity cases the department identifies some candidate they want and they offer the position without a search.

d. I hate imagining you lying there alone, in some godforsaken hospital.

e. I want to get some book about St. Petersburg because we are going there soon.

f. If Ben solves some problem from this list Mr. Koens will praise him.

Just like $a(n)$ indefinites, some DPs have 'free upaward' scope. Thus, [26]

[26] Keith decided to buy every album that was published by some famous Hungarian photographer.

has three interpretations: (i) the indefinite has widest scope if the sentence describes a situation in which Keith decided to concentrate on a particular photographer, say, Lucien Hervé; (ii) the indefinite has intermediate scope if the situation described is one in which Keith decided to choose one photographer from the group of famous Hungarian photographers known to him (Moholy-Nagy, Brassai, Capa or Hervé) but has not yet made up his mind which; (iii) the indefinite has narrow scope if it describes a situation in which Keith decided to buy every album published by each of these photographers. ${ }^{8}$

Some differs from $a(n)$, however, in that it is compatible only with existential force. Thus, some may not be interpreted generically in sentences like [27] (as noted by Langacker 1991), and is not available for unselective binding.

[27] $\mathrm{A} /$ * Some seagull lays eggs in the sand.

The question that arises is how to capture this stubborn existential force. One possibility is to treat some as a quantificational DP, just like every or most, but involving an existential quantifier. Under this view, however, the freedom of upward scope of some DPs exemplified in [26] remains a mystery, given the well-known limitations in the upward scope possibilities of quantificational DPs. Another option is to 
treat some as introducing a Reinhart-style choice-functional variable which is freely bound by existential quantifiers inserted freely in the semantic representation. The explanatory gain concerning the scopal properties of choice-functional variables is rather modest, however, since we have to stipulate the special nature of the binding of such variables. (See Geurts 2000 for relevant discussion.)

In line with the proposal for dependent indefinites made above, one way of capturing the existential nature of some indefinites is to assume that the variable they introduce is subject to the functional constraint in [28]:

\section{[28] Existential constraint}

If a variable $x_{n}$ is introduced by some its evaluation functions may not be coindexed with it.

Under this view some is non-quantificational, just like $a(n)$ and simply introduces a variable, and therefore some is not expected to have the scopal properties typical of quantificational DPs. The variable is, however, subject to the functional constraint in [28] which rules out interpretations which make this variable cover its value set.

The constraint in [28] prevents the variable introduced by some from being unselectively bound as well as from being within the immediate scope of negation since these interpretations involve the variable being evaluated by a set of co-indexed functions. Evaluation fates that are consistent with [28] are: (i) being given values by the initial function $f$ (scopal specificity); (ii) being given values by functions $\mathrm{G}_{m}$, where $m \neq n$ (co-variation); (iii) being evaluated relative to a world or worlds different from the world of evaluation of the discourse. The fact that some indefinites may be scopally specific, may co-vary and may occur within the scope of modals, intensional predicates and conditionals is thus accounted for.

In the rest of this subsection I examine in more detail the interaction of some DPs and negation. As just mentioned, [28] predicts the PPI status of some DPs, and therefore the fact that some in [29] may only be interpreted as having wide scope relative to negation:

[29] Mary didn't buy some apartment in San Francisco when she could have afforded it and now it's too late.

Interestingly, [28] alo predicts that some DPs may occur embedded under negation, as noted in Ladusaw 1980 and exemplified in [30].

[30] Mary doesn't think that Sue read some recent article on this problem.

The indefinite here occurs within a file which itself is subordinate to the negated one, as schematized in [31],

$$
[31]\left[\rightarrow\left[\ldots\left[\ldots x_{n 2} \ldots\right]_{\phi^{13}}\right]_{\phi^{\prime}}\right] \phi
$$

and therefore its index will not appear on the functions introduced by the negative operator.

It has long been noted, however, that there are several environments where some DPs may occur apparently in the immediate scope of negation, in violation of [28]. 
Szabolcsi 2001 summarizes these cases as involving either denial, as exemplified in [32], or as involving contexts which otherwise license negative polarity items, as exemplified in [33]. (The examples are adapted to contain a some DP rather than Szabolcsi's someone.)

[32] A: He found some mistake.

B: Wrong! He DIDn't/DID NOT find some mistake.

[33] a. If we don't call some neighbor to help, we are doomed.

b. Every boy who didn't call some friend to help was in trouble.

c. Only John didn't call some friend to help.

d. Few boys didn't call some friend when they saw the difficulty they were in.

e. Few boys thought that John didn't call some friend.

We can add to these the real-life example in [34]:

[34] They want to make sure there isn't some $\mathrm{Al}$ Qaeda leader waiting in the wings to take over after Bin Laden is eliminated. (NPR, Morning Edition, 12/10/01).

Szabolcsi's account of [33] rests on assuming that some bears two negative polarity features that become active only in a negative environment. Once active, each has to be 'neutralized', one by the negative in whose scope some finds itself, and the other by the NPI licensing operator that some scopes under.

This line of analysis does not extend to [32] and remains somewhat stipulative. An alternative that holds the promise of extending to both denials and the cases in [33] would be to refine our understanding of negation rather than complicate our analysis of some. A direction worth exploring is to attempt to reduce all cases in which some introduces a variable in the domain of a negated file $\psi$ to special cases of negation, cases where alternatives in which $\psi$ holds survive in the output context. Denying $\psi$, as in B's utterance in [32] would fall under this characterization if we assume the articulated view of context proposed in Gunlogson 2001 according to which the common ground of a conversation is split into two files, $\phi_{A}$ and $\phi_{B}$, one for each participant. Possibilities in which $\psi$ holds survive in the output context because after the exchange in [32], $\mathrm{c}\left(\phi^{\prime} A\right)$ contains only possibilities that satisfy $\psi$, while $\mathrm{c}\left(\phi I_{B}\right)$ does not. The task of showing that in each of the examples in [33] relevant alternatives in which $\psi$ holds survive in the output context is neither trivial nor impossible. The connection with NPI licensing environments could be captured by exploiting the fact that what is involved in these examples is a sentence $S$ occurring in an environment where alternatives in which the opposite of $S$ holds are salient and survive in the output context. If $S$ is positive, we expect this property to connect to NPI licensing; if S is negative, we expect it to connect to PPI licensing. Developing these suggestions is, however, beyond the scope of this paper.

I conclude that some DPs differ from their unmarked $a(n)$ DP counterparts in being stubbornly existential. The functional condition in [28] captures this property 
without having to treat some DPs as quantificational, and thereby preserving a unified account of the freedom of upward scope of $a(n)$ and some DPs. The condition also predicts the lack of generic readings and the PPI-hood of some DPs. Accepting it makes us look for a solution to the problem posed by the counterexamples just discussed in the realm of negation rather than in the realm of indefinites. The hope is that a deeper understanding of NPI licensing in the contexts exemplified above will shed light on the partial overlap between NPIs and PPIs.

\subsection{Unidentified variables}

Capturing the limits on the quantificational force of some does not account for the full range of its peculiarities. In this subsection I attempt to capture the intuition that using some is connected to the fact that the value one chooses for the variable introduced by it is underspecified in some manner. Note that destressing some or adding or other stresses this property, forcing narrow scope relative to want in [35]:

[35] I want sm book (or other) about St. Petersburg.

Note, however, that destressed or modified some does not have to be within a modal context (unlike free-choice any):

[36] Sm guy (or other) called this afternoon and asked for you.

The unstressed version and the modification by or other is associated with the fact that the identity of the choice of value for the variable introduced by some is immaterial, in [35] because the ordering relation on alternatives involved in the semantics of want is insensitive to which book the speaker gets, in [36] because the context provides no distinguishing features of the guy who called. In [37] we see an example of 'epsitemic non-specificity' openly confessed: the speaker does not have enough information to identify the verifying value of the variable introduced by some:

[37] There's some mistake here somewhere but I don't know where.

Sentence [38] exemplifies the derogatory flavor that some DPs may have, connected, I claim, to the fact that the verifying value is not to be distinguished from others. ${ }^{9}$

[38] Marc wrote some paper (or other) on indefinites and now he considers himself a specialist.

In [39] we see some used in a situation where its verifying value is a particular entity (one of the streets enumerated in the sentence), therefore it is in principle identifiable, but the context does not provide the necessary information to narrow down the choice to a single street.

[39] In his deteriorated mental state, he rented a room, a chamber, a little more than a hole on some disreputable street - on Kleine Gröpel Grube, on Engelswisch or on An der Mauer - and then slunk into his hideway to eat his pastries. (Th. Mann, Buddenbrooks, translated by John E. Woods, p. 399) 
In [40], from Emily Dickinson, we have an example of some whose verifying value is not identifiable in principle:

[40] Our lives are Swiss -/ So still, so cool,/ Till some odd afternoon,/ The Alps neglect their curtains,/ And we look farther on.

In [41] we see that some DPs may not occur as objects of a measure phrase:

[41] *Marc walked some mile before he stopped to rest.

The next example, from Israel (ms), illustrates a 'no imminent identification' requirement: if a speaker chooses some it is judged as marked for her to immediately go ahead and identify the verifying value in the subsequent discourse without a signal of change of mind, or in the absence of a request from the audience for more information.

[42] a. Susan rented some movie for us to watch yesterday.

b. \#It was The Maltese Falcon.

The next two examples show that the use of some is not appropriate in cases where the identification of the verifying value is pragmatically irrelevant:

a. Oh look! There's a/\#some fly in my soup!

b. A/\# some cab will be waiting for you at the airport.

We normally don't care which particular fly is in our soup once the soup is found not to be fly-free, and, similarly, we don't care which particular cab will be waiting for us at the airport, as long as we are assured we will not be left on our own.

The empirical generalization that captures these characteristics is formulated in [44]. (See Abusch and Rooth 1997 for a similar characterization of indefinites modified by epistemic adjectives such as undisclosed.)

[44] After an expression containing some is added to a file $\phi$, the possibilities of the new context $c\left(\phi^{\prime}\right)$ are required to vary with respect to what value is assigned to the variable introduced by some.

In order to make [44] more precise I first define in [45] the notion of identified variables:

\section{[45] Identified variables}

a. An independent variable $x_{n}$ occurring in $\phi$ is identified in $\mathrm{c}(\phi)$ iff there is an $a \in \mathrm{D}$ such that for every possiblity $\langle w, f\rangle \in \mathrm{c}(\phi)$, if $h$ is an evaluation function of $x_{n}$ in $\langle w, f\rangle, h\left(x_{n}\right)=a$.

b. A dependent variable $x_{n}$ occurring in $\phi$ is identified in $\mathrm{c}(\phi)$ iff for every parameter $v$ of $x_{n}$ there is an $a \in \mathrm{D}$ such that for every possibility $\langle w, f\rangle \in \mathrm{c}(\phi)$, if $h^{v}$ is an evaluation function of $x_{n}$ in $\langle w, f\rangle, h^{v}\left(x_{n}\right)=a$. 
(The values of the subscript on an assignment function are the the values of the variable $x_{n}$ depends on.) An independent variable is identified in a context iff it is assigned the same value in all the possibilities of the context. A dependent variable is identified in a context iff it is identified relative to each of its parameters. ${ }^{10} \mathrm{I}$ define now unidentified variables relative to a context in [46]:

\section{[46] Unidentified variables}

a. An independent variable $x_{n}$ occurring in $\phi$ is unidentified in $\mathrm{c}(\phi)$ iff there are at least two elements $a_{1}$ and $a_{2} \in \mathrm{D}$ such that there are at least two possibilities, $\langle w, f\rangle,\langle w \prime, f \prime\rangle \in \mathrm{c}(\phi)$, and if $h$ is an evaluation function of $x_{n}$ in $\langle w, f\rangle, h\left(x_{n}\right)=a_{1}$, and if $h$ is an evaluation function of $x_{n}$ in $\langle w \prime, f \prime\rangle, h\left(x_{n}\right)=a_{2}$.

b. A dependent variable $x_{n}$ occurring in $\phi$ is unidentified in $\mathrm{c}(\phi)$ iff there are at least two elements $a_{1}$ and $a_{2} \in \mathrm{D}$ such that there are at least two possibilities, $\langle w, f\rangle,\langle w \prime, f \prime\rangle \in \mathrm{c}(\phi)$, and if $h^{v}$ is an evaluation function of $x_{n}$ in $\langle w, f\rangle, h^{v}\left(x_{n}\right)=a_{1}$, and if $h^{v}$ is an evaluation function of $x_{n}$ in $\langle w \prime, f \prime\rangle, h^{v}\left(x_{n}\right)=a_{2}$.

A variable is unidentified in a context iff the values it is assigned in various possibilities in that context are not necessarily the same. A variable may be unidentified either because there is not enough information in the current context to identify it or because it is unidentifiable in principle. The former situation obtains either because the speaker does not possess the required information to identify the variable or because she judges it irrelevant to the purposes of the conversation to provide it. A variable is unidentifiable in principle if it has narrow scope relative to dispositionals, certain modals or conditionals. We will come back to unidentifiable variables in Section 5.

Turning now back to some, a first attempt is to suggest that it is lexically specified to impose the condition in [47] on the variable it introduces because

\section{[47] Unidentified variable constraint}

If $x_{n}$ is introduced by some in an expression that is added to a file $\phi$ resulting in a file $\phi^{\prime}, x_{n}$ must be unidentified in $\mathrm{c}\left(\phi^{\prime}\right)$.

This is an output context constraint, since it constrains the status of the variable in the output context. Note that the existential constraint in [28] could be made to follow from [47] were we to rule out contexts were a variable has to cover a singleton set. If such cases are ruled out, variables evaluated by a set of co-indexed functions could never be unidentified: there will be no possibility in which the variable is assigned a single value.

If we want to capture the 'no imminent identification' restriction observed by Israel, [47] has to be strengthened so as to require the variable in question to stay unidentified in the contexts that the speaker projects as being the future contexts the conversation is to reach. In order to do this, I first take from Gunlogson 2001 the notion of the reduction set of a context $c(\phi), \mathcal{R}(\phi)$. (See also Dekker 1993) This is 
the set of contexts that can be reached from $\mathrm{c}(\phi)$ by increasing information. I define the reduction set here based on an accessibility relation $\mathrm{R}$ between contexts which is defined in [48]:

[48] $\mathrm{c}(\phi) \mathrm{R} \mathrm{c}(\phi)$ iff for every $\langle w, f\rangle \in \mathrm{c}(\phi)$ there is a possibility $\langle w, f\rangle \in \mathrm{c}(\phi)$ such that $f \prime$ is a possibly trivial extension of $f$.

The reduction set $\mathcal{R}(\phi)$ of a context $\mathrm{c}(\phi)$ is the set of contexts $\mathrm{R}$-accessible from $\mathrm{c}(\phi)$. Note that if $\mathrm{c}(\phi)$ is R-accessible to $\mathrm{c}(\phi)$ and the context set of $\mathrm{c}(\phi)$ is not empty, $\mathrm{Wc}(\phi) \subseteq \mathrm{Wc}(\phi)$ and $\mathrm{Wc}(\phi) \neq \emptyset$.

The notion that we need, that of the subset of accessible contexts that the speaker projects as future contexts, can be defined as a proper subset $\mathcal{P}(\phi)$ of $\mathcal{R}(\phi)$. We can now talk of stable lack of identification in case a variable is unidentified in all the contexts in $\mathcal{P}(\phi)$. I suggest then that some imposes on the variable it introduces the output context constraint in [49]:

\section{[49] Stable lack of identification}

A variable introduced by some must be unidentified in every $c^{\prime}\left(\phi^{\prime}\right) \in \mathcal{P}\left(\phi^{\prime}\right)$.

Let us consider now the consequences [49] has concerning the distribution of some DPs. Note that the impossibility of these DPs to occur as measure phrases follows, since measure units are unique relative to a model. Some DPs are not good as existence assertions but are perfectly suited to indicate epistemic non-specificity. The contrast between [50a], which indicates that the speaker doesn't know (or won't tell) what the reason for Mary's depression is, and [50b], which indicates that Mary's depression is not without a reason, is not surprising.

[50] a. Mary was depressed for some reason (and could not go to the party).

b. Mary was depressed for a reason.

Some DPs, just like their unmarked sisters, are compatible with both dependent and independent interpretations and therefore the ambiguity of the examples in [25] is predicted. They are, however, less precise because of the stable lack of identification condition. This, I suggest, is responsible for the use of some as approximatively, exemplified in [51].

[51] There were some three hundred people at the rally.

Plural some and mass some share the lack of identification property that singular some has. They require the existence of a number or quantity from the value set and what is unidentified is the exact quantity/number involved. We predict that Ds associated with lack of identification in other languages may also have such uses. Romanian vreun and French quelque appear to confirm this prediction. (cf. Farkas (to appear)).

The fact that some is not felicitous in cases where identification is pragmatically irrelevant (as in [43]) is also unsurprising: why use a marked form to indicate that a variable will stay unidentified when there is no reason to expect that it should be? 
To sum up, it has been claimed here that some is marked relative to $a(n)$ along two closely related dimensions: (i) quantificational force and (ii) marked lack of present and future identification. The fact that some DPs occur in a subset of the environments in which we find $a(n)$ follows from these two properties. In the next section we see that an output context condition involving identification is crucial for accounting for the distribution and interpretation of $a$ certain DPs.

\section{A certain indefinites}

We first examine briefly the distribution of a certain indefinites, and then propose a constraint that accounts for it.

A certain DPs love to be scopally specific, as in [52],

[52] I spoke to a certain high official yesterday, who assured me that everything is all right.

but we have known at least since Hintikka's 1986 example, [53], that they may be dependent.

[53] Every man forgot a certain date: his wife's birthday.

In [54] we see that there is no need for a contextually salient function from the domain of the universal to that of the indefinite for a dependent reading of a certain to be possible.

[54] Every student chose a certain poem by Emily Dickinson and analyzed it.

It is the case, however, that dependent a certain, unlike dependent $a(n)$ or some, requires there to be a non-random choice of value for the variable introduced by it relative to its evaluation parameter. This explains the strangeness of [55]: ${ }^{11}$

[55] \#Everybody will choose a certain poem at random.

Just like some, a certain must be existential. As shown by the unambiguous [56], the variable introduced by a certain may not be interpreted generically and may not occur within the immediate scope of negation.

[56] a. A certain seagull lays eggs in the sand.

b. Mary didn't buy a certain apartment in San Fancisco when she could afford it and now it is too late.

A certain DPs are special, however, in that they resist narrow scope interpretations relative to some (but not all) modal expressions. Thus, in [57], the indefinite is necessarily interpreted as having wide scope.

[57] a. I hate imagining you lying there alone in a certain hospital.

b. If Ben solves a certain problem from this list Mr. Koens will praise him. 
Concluding that a certain DPs must be interpreted relative to the world of evaluation $w$ would be too hasty, however, given that the examples in [58] are scopally ambiguous:

a. I dreamt that I had to catch a certain unicorn.

b. Mary believes that a certain unicorn is ravaging her garden.

We conclude that a certain DPs may occur within the scope of weak intensional predicates such as dream, believe and think but not within the scope of conditionals or dispositionals.

In [59] we see that an indefinite in the complement of want is in principle three way ambiguous:

[59] Poor delusional Mary! She wants to catch a/some/a certain unicorn (she thinks has been ravaging her garden).

(i) The indefinite may have widest scope, in which case unicorns are assumed to exist in the world of the discourse; (ii) the indefinite may have 'intermediary' scope (strengthened by the material in parentheses): unicorns exist only in Mary's epistemic alternatives, and she has a particular unicorn in mind that she wants to catch; (iii) the indefinite may have narrowest scope, in which case Mary's wish is satisfied no matter which (imaginary) unicorn she catches. Crucially for us, the variant with a certain is compatible with (i) and (ii) but not (iii).

The correct account of a certain has to capture these modal peculiarities, the non-randomness requirement on dependent a certain, as well as the differences and similarities between a certain and the other indefinites in its paradigm. My proposal is that a certain involves a special output context constraint requiring the variable it introduces to be identifiable based on a non-trivial identifying property. ${ }^{12}$ When using a certain, one signals that there is an entity that is the right choice of value for the variable a certain introduces. While neither the current nor future contexts necessarily provide the information that would render the variable identified, there must be accessible contexts where the variable is identified.

In order to be able to formulate this constraint I define first the notion of identifiable variable in [60]:

\section{[60] Identifiable variable}

A variable $x_{n}$ occurring in $\mathrm{c}(\phi)$ is identifiable in $\mathrm{c}(\phi)$ iff there is a $\mathrm{c}(\phi) \in$ $\mathcal{R}(\phi)$ such that $x_{n}$ is identified in $\mathrm{c}(\phi)$.

A variable is identifiable in a context if the context can in principle be extended to one where the variable is identified. There is no requirement that anybody possess identifying knowledge or that the variable be identified in the immediate future of the conversation, but only that it be in principle identifiable. If a variable is not identifiable, it is unidentifiable. If a variable is unidentifiable, there is no accessible context in which it is identified, i.e, there is no accessible context in which the variable is given a single value in all possibilities in that context.

The notion of a (non-trivial) identifying property for a variable relative to a context is defined in [61]: 
[61] Identifying property of $x_{n}$

$\mathrm{P}$ is an identifying property of $x_{n}$ in $\mathrm{c}(\phi)$ iff $x_{n}$ is identified in $\mathrm{c}(\phi)$ and for any $h^{(v)}$ that assigns a value to $x_{n}$ in $\mathrm{c}(\phi), h^{(v)}\left(x_{n}\right)=\iota x P(x)$.

The constraint imposed by a certain on the variable it introduces is formulated in [62]:

\section{[62] Identifiable variable constraint}

If a variable $x_{n}$ is introduced by a certain in $\phi$ resulting in $\phi \prime$, (i) $x_{n}$ may not be identified in $\mathrm{c}\left(\phi^{\prime}\right)$ and (ii) there must be a non-trivial identifying property $\mathrm{P}$ relative to some $\mathrm{c}\left(\phi^{\prime}\right)$ accessible to $\mathrm{c}(\phi \prime)$.

So far a certain was treated as a complex D. A compositional analysis could be attempted by taking the adjective certain to contribute the restrictive condition $\operatorname{certain}\left(x_{n}\right)$ on the variable introduced by $a$ and assume that the denotation of the adjective certain is the set of identifying properties. An immediate problem that arises is the explanation of why certain can only occur with $a$, and therefore why * some certain, for instance, is ungrammatical. The task is not trivial, since we saw that some DPs are compatible with the variable being identifiable. In order to explain the incompatibility of some with certain one would have to require the former to impose a constraint against the existence of an identifying property for the variable it introduces relative to accessible contexts, a constraint that appears too strong. Thus, a compositional account of a certain is close, but still out of reach.

I turn now to examining some of the consequences of [62] relative to the distribution of a certain DPs. First, note that a variable $x_{n}$ occurring in $\mathrm{c}(\phi)$ is unidentifiable in $c(\phi)$ if it is required to be given several values in every possibility in $c(\phi)$. This happens in case the variable is interpreted by a set of functions coindexed with it, i.e., if it is made to cover its domain. This is so because in every context accessible to $\mathrm{c}(\phi)$ where the value set of $x_{n}$ is not necessarily singleton, $x_{n}$ is given multiple values. (Recall that we assume that if a variable is made to cover its value set, contexts in which all possibilities involve a singleton value set for the variable in question are ruled out.) It also happens in case a variable is given values by assignment functions across a set of worlds that are required to differ with respect to the value they assign to it. In this case, in every possibility in $c(\phi)$ the variable is given multiple values.

There are three immediate consequences of [62]. (i) A certain indefinites are predicted to be existential in the sense of being incompatible with interpretations that make them cover their value sets. The fact that these indefinites have no generic readings, are not available for unselective binding and my not occur in the immediate scope of negation is accounted for. (ii) A certain indefinites are predicted not to occur within the scope of conditionals, dispositionals or under the narrowest interpretation in the complement of want. This is so because such variables must be assigned different values at different worlds and therefore their interpretation is not compatible with [62]. (See subsection 5.2. for details.) (iii) The possibility of $a$ certain DPs to be dependent is predicted, since dependency is not incompatible with identifiability. The non-random choice of value for dependent variables introduced 
by a certain is due to the requirement of the existence of the identifying property.

\subsection{A certain and Weak Cross Over}

We now take a brief look at the proposal made in Kratzer 1998 to treat a certain as contributing a choice-functional variable, under the assumption that such variables can be bound only contextually (or, alternatively, by text-level existential closure). Dependent interpretations of a certain DPs are accounted for by assuming that choice functions may involve implicit arguments that are bound independently of the choice functional variable. The effect of dependency is created by having such an implicit argument be bound by the universal the indefinite appears to be dependent on. In defense of this analysis, Chierchia 2001 brings up the fact that in [63], a certain engineer cannot have narrow scope relative to every building.

[63] A certain/an engineer inspected every building

The lack of ambiguity here is attributable to $\mathrm{W}$ (eak) $\mathrm{C}$ (ross) $\mathrm{O}$ (ver) under the assumption that the dependent interpretation would involve every buidling crossing over the co-referential implicit argument of a certain. It turns out, however, that in less bland examples, a certain DPs may in fact have narrow scope relative to a universal they c-command:

[64] a. A certain carefully selected location will serve as backdrop to every presidential speech.

b. A certain appropriately specialized lawyer will deal with every aspect of this complicated case.

c. A certain member of the faculty phoned every applicant in an attempt to increase our incoming class.

Next, note that having a certain analyzed as introducing a Kratzer-style choice functional variable would not explain the complexities of the interpretation of these DPs in modal environments without extra stipulations on the binding properties of such variables. The next subsection is devoted to working out some of the details involved in an account of the interpretation of a certain in such environments. Presenting a fuller picture would take us further into intensional semantics than we can go here.

\subsection{A certain in intensional environments}

We first consider why [62] does not rule out a certain DPs from being interpreted within the scope of predicates like think and dream and then turn to scope effects in conditionals and in the complement of want.

Epistemic predicates (think, believe, know) require their complements to be interpreted relative to the epistemic base of the referent of their subject. An epistemic base $\mathrm{E}_{a, w}$ is the set of worlds associated by an accessibility relation $\mathrm{E}$ in $\mathrm{A}$ to an individual $a$ and a world $w$. Epistemic bases are like context sets in that they contain 
worlds consistent with what $w$ is taken to be (as far as the conversation is concerned, in the case of the latter, and as far as $a$ is concerned in the case of the former). Sets of worlds having this property will be called centered. I assume that every centered set of worlds $W_{c}$ has two properties: (i) consistency, defined in [65] and (ii) non-empty domain intersection, defined in [66].

[65] Consistency

$\mathrm{W}_{c} \neq \emptyset$

[66] Non-empty domain intersection

For every $\mathrm{W}_{c}, \bigcap_{w \in W_{c}} D_{w} \neq \emptyset$.

Turning now to [67], it is generally assumed that in order for it to be true in $w$,

[67] John thinks that [a unicorn is ravaging his garden ] $S^{\prime}$.

S ' must be true in all the worlds $w \prime \in \mathrm{E}_{j, w}$, where $j$ is the individual named by John. The complement of epistemic verbs then has to be true in the worlds of the epistemic base associated to its subject, which is a centered set. I assume that the complements of fictional verbs such as dream or those of verbs of saying such as say, claim, declare share with epistemic verbs the property of having to be true in a set of centered worlds. The non-empty intersection of the domains of the worlds in a centered set will be called the domain of the set in question.

The effect of adding [67] to a context $\mathrm{c}(\phi)$ resulting in an output context $\mathrm{c}(\phi \prime)$ is to rule out from the possibilities in the input context all those in which the complement is not true in John's epistemic base relative to the relevant world. The output context is characterized as in [68], where $\psi$ is the semantic representation of S/ and $\mathrm{c}(\psi)$ is the set of world-function pairs that satisfy it in $\mathbf{M}$.

[68] $\mathrm{c}(\phi \prime)=\left\{\langle w, f\rangle \in \mathrm{c}(\phi)\right.$ : for every $w^{\prime} \in \mathrm{E}_{j, w}$, there is an extension $f \prime$ of $f$ such that $\langle w \prime, f \prime\rangle \in \mathrm{c}(\psi)\}$

Common to epistemic and fictional predicates, as well as to verbs of saying, is that their complement is added to a centered set of worlds in the same way in which declarative sentences are added to a context. In both cases the effect is to shrink a centered set by removing from it worlds in which the sentence is not true.

If a variable $x_{n}$ has wide scope relative to think it is given values by $f$ from $\mathrm{D}_{w}$. If it has narrow scope, it is given values by $f \prime$ from the domains of the worlds in $\mathrm{E}_{j, w}$. Crucially, however, in neither case is the variable required to be assigned multiple values in all contexts that are accessible to $c(\phi \prime)$. If the variable has wide scope, contexts where the variable is identified are not ruled out: there are contexts in the reduction set of $\mathrm{c}\left(\phi^{\prime}\right)$ where $x_{n}$ is identified, and therefore $x_{n}$ is identifiable. Recall that the contexts in the reduction set of $\mathrm{c}\left(\phi^{\prime}\right)$ are more informative and therefore they involve contexts whose context sets are subsets of $\mathrm{Wc}(\phi \prime)$. One such context is the omniscient one, where the only element of the context set is $w$.

If the variable has narrow scope, it remains identifiable. More informative contexts may contain more information concerning what John thinks and in such contexts $x_{n}$ may be identified. This is so because more informative contexts may involve adding information concerning John's epistemic base and therefore shrinking 
the worlds compatible with what John knows in every world of the context in question. Complete epistemic bases, where the set of worlds compatible with what John knows is a singleton are not ruled out. Since nothing rules out more informative contexts in which $x_{n}$ is identified, $x_{n}$ is identifiable. The same is true of the complements of fictional verbs and verbs of saying. In their cases too, there are accessible contexts where the bases they introduce shrink enough to have the variable in their scope identified. We thus predict that [69] does not necessarily commit its speaker to a belief in unicorns:

[69] John thought/dreamt/said/ that a certain unicorn was ravaging his garden.

Let us now consider indefinites occurring in the antecedent of a conditional, as in [70].

[70] If Ben solves a/some/a certain problem from this list Mr. Koens will praise him.

This sentence is true in a world $w$ iff for every $w /$ that is close to $w$ and in which the antecedent is true, the consequent is true in $w /$. Assuming that [70] is added to a context $\mathrm{c}(\phi)$, the resulting context $\mathrm{c}(\phi \prime)$ is defined as in [71], where $\psi$ and $\psi \prime$ are the semantic representations of the antecedent and the consequent respectively.

[71] $\mathrm{c}(\phi \prime)=\{\langle w, f\rangle \in \mathrm{c}(\phi)$ : for every $w /$ close to $w$ such that there is an extension $f \prime$ of $f$ and $\left\langle w \prime, f^{\prime}\right\rangle \in \mathrm{c}(\psi)$, there is an extension $f^{\prime \prime}$ of $f \prime$ such that $\left\langle w \prime, f^{\prime \prime}\right\rangle \in$ $\left.\mathrm{c}\left(\psi^{\prime}\right)\right\}$

I assume that conditionals carry the presupposition that the set of worlds established on the basis of the antecedent is non-empty and non-singleton, i.e., they are felicitous only in contexts that meet this condition.

When a variable introduced in the antecedent has wide scope relative to the conditional, it is evaluated by $f$ relative to $\mathrm{D}_{w}$ and is identifiable like all scopally specific variables. We correctly predict therefore that the indefinite in [70] may have wide scope relative to the conditional, describing a situation in which $\mathrm{Mr}$. Koens's praise is not easy to earn. In more informative accessible contexts we find out which problem is the one that would elicit it. When, on the other hand, such a variable has narrow scope relative to the conditional, values for $x_{n}$ are given by $f^{\prime}$ from $D_{w}$. The set of worlds established by the antecedent will contain a world for every problem on the list. No further information can narrow down the choice of value for $x_{n}$ since solving any of the problems on the list elicits Mr. Koens's praise. The problems must co-vary with the worlds, and since the worlds cannot be a singleton set in all possibilities in a context, the variable will not be identified in any. Our analysis correctly predicts that $a(n)$ and some may have narrow scope relative to the conditional but a certain may not.

Let us consider now indefinites in the complement of want, exemplified in [72]:

[72] John wants to catch a/some/a certain unicorn. 
I follow Heim 1992 in assuming that want requires a particular ordering relation on the worlds in the epistemic base of its subject. The sentence in [72] is true in a world $w$ iff there is a partial ordering (or preference) relation on the worlds in $\mathrm{E}_{j, w}$ such that every $w / \in \mathrm{E}_{j, w}$ in which the complement of want is true is ordered higher than closest worlds in which the complement is not true. The output context $\mathrm{c}\left(\phi^{\prime}\right)$ that results from adding [72] to an input context $\mathrm{c}(\phi)$ is defined in [73], where $\psi$, is the semantic representation of the complement of want:

[73] $\mathrm{c}(\phi \prime)=\left\{\langle w, f\rangle \in \mathrm{c}(\phi)\right.$ : for every $w / \in \mathrm{E}_{j, w}$ such that there is an extension $f \prime$ of $f$ and $\langle w \prime, f \prime\rangle \in \mathrm{c}(\psi), w \prime\rangle w^{\prime \prime}$, where $w^{\prime \prime}$ is maximally similar to $w^{\prime}$ and $\left.w^{\prime \prime} \in \mathrm{E}_{j, w}-\mathrm{Wc}(\psi)\right\}$.

The verb want shares with epistemic predicates the fact that the epistemic base of its subject is relevant to establishing the truth conditions of sentences whose main verb it is. Unlike those predicates, however, the effect of the complement on the epistemic base is different. The role of the complement of want is similar to that of the antecedent of a conditional because it is used to define a set of worlds: all the worlds (within a given set) in which the complement is true must be ordered higher than those in which it is not.

The evaluation possibilities of a variable introduced by an indefinite occurring in the complement of want are the following: (i) The variable may be given values by $f$ relative to $\mathrm{D}_{w}$ (widest scope). (ii) The variable may be given values by $f \prime$ relative to $\mathrm{D}_{j, w}$, the domain of John's epistemic base in $w$ (intermediary scope). (iii) The variable may be given values by $f \prime$ relative to $\mathrm{D}_{w^{\prime}}$ (narrowest scope). In the first case the variable is identifiable, as all scopally specific variables are. In the second case the variable is identifiable too because its value is constant across the worlds $w \prime$. Under this interpretation, within every possibility in $\mathrm{c}(\phi)$ (and within every possibility in the contexts $\mathrm{R}$-accessible from it), worlds that are required to be preferred relative to that possibility involve John catching the same unicorn. The only difference between (i) and (ii) is that in this case the unicorn is chosen among the entities John believes exist. (iii) Finally, the variable may be given values by $f \prime$ relative to $w /$. In this case, all the worlds in $\mathrm{E}_{j, w}$ in which John catches some unicorn or other are preferred to those in which he does not. The preference relation now is insensitive to which unicorn John catches and the values assigned to the variable introduced by the indefinite co-vary with $w /$. More informative contexts cannot single out a unique unicorn that John has to catch in order to be in a preferred world. There is no accessible context in which the variable introduced by the indefinite is given the same value across all possibilities in the context, and therefore under this interpretation the variable is not identifiable. The constraint in [62] is compatible with the first two evaluations but not the third and therefore a certain cannot be given a narrowest scope interpretation. ${ }^{13}$ The difference between want and conditionals is that the former makes possible the interpretation of an indefinite within its complement relative to the epistemic domain of the subject, while conditionals do not involve this extra layer. 


\section{Conclusion}

We have suggested here that the three Ds examined differ with respect to constraints they impose on the variable they introduce. The $\mathrm{D} a(n)$ is unmarked, in that it imposes no constraints whatsoever. Some is marked in that it imposes a functional constraint and an output context constraint requiring the variable to be unidentified in projected contexts. A certain is marked in that it imposes an output constraint requiring the variable it introduces to be identifiable on the basis of a property. This latter constraint turns out to be more restrictive than the two constraints imposed by some and therefore the concentric distribution of the three Ds is accounted for.

Treating $a(n)$ as the unmarked singular D may help explain why it and none of the others is used in situations where a DP does not introduce a variable, as in the case of predicative DPs, exemplified in [74], or in cases where the DP is propertydenoting, as in existence statements, or as objects of prevent. ${ }^{14}$

[74] Mary is a/*some/*a certain doctor.

[75] a. There is a/*some/*a certain God afterall!

b. Mary has a/*some/*a certain brother.

[76] Negotiations prevented a/*some/*a certain war.

I assume that in these cases the presence of the D is syntactically motivated and that these DPs do not involve the introduction of a variable. Choosing the least marked D as the default D is explicable in optimality-theoretic flavored terms: the only property of $a(n)$ one has to disregard here is that of introducing a variable. In the case of the other Ds we would have to disregard their extra constraints as well.

If the approach suggested here is on the right track it would allow us to capture the details of the distribution and interpretation of other Ds in English and elsewhere. Further work would, hopefully, lead to a better understanding of the type of constraints Ds encode and eventually, to a better understanding of their cross-linguistic typology. Particularly easy to characterize in present terms is the constraint imposed by another, brought to my attention by Bill Ladusaw. The constraint is that the variable it introduces must be given a value different from that given to a previously introduced variable. This is an input context constraint in the sense that it requires the prior existence of a variable. Accounting for indefinite this requires a different type of constraint, one involving topicality or saliency. An interesting open question is what other constraints are needed. In particular, with respect to identifiability, the discussion above suggests that a possible constraint would be one requiring the variable introduced to be unidentifiable. Whether such determiners actually exist remains to be seen. 
Notes

*Various parts of this work were presented during a seminar in Winter 2001, at Santa Cruz, at a workshop on choice functions, August 2001, in Helsinki, at the Paris Colloquium on Syntax and Semantics (CSSP), October 2001, at a DiP Colloqium, April 2002, in Amsterdam, and at Chicago, in May 2002. I wish to thank audiences at these events, as well as at SALT 12, in San Diego, for helpful questions and comments. I am grateful to Christine Gunlogson for discussions on matters relevant to this paper, and to Jaye Padgett and Benjamin Farkas for being patient native consultants. An earlier version of the section on some will appear in the proceedings of the CSSP conference, and an earlier incarnation of this paper appeared as On some Indefinites in English in K. von Heusinger, R. Kempson and W. Meyer-Viol (eds.), Proceedings of the Workshop "Choice Functions and Natural Language Semantics", Arbeitsbapier 110.FB Sprachwissenschaft, U. Konstanz, pp. 35-62.

1. Issues of topicality and saliency would become crucial were we to extend our attention to indefinite this, first discussed in Prince 1981.

2. One important advantage is that assignments outside the value set are irrelevant for satisfaction and truth conditions. A related advantage is that material implication can be dispensed with when giving the semantics of universally quantified expressions. Under the assumption that natural language conditionals are not to be treated as material implication either, one circumvents the problem discussed by Heim 1982 and Reinhart 1997 concerning the 'in situ' interpretation of wide scope indefinites occurring in the antecedents of conditionals.

3. In Heim 1982 auxiliary files exist only as part of the process of interpretation, as substeps in complex satisfaction conditions.

4. I assume that semantically relevant number features are responsible for semantic singularlity/plurality effects. Since we are looking only at singular Ds, details concerning the interpretation of number features can and will be ignored here.

5. I will not be concerned here with the question of what determines the choice between the two possible interpretations for the indefinite.

6. The fact that dependent indefinites may not co-vary with a generic indefinite either, shown by the ungrammaticality of *Egy sirálynak van egy-egy csöre 'A seagull has a-a beak', is due to an additional constraint on reduplicated indefinites requiring the value set of the variable they depend on to be well-delimited in some sense. While reduplicated indefinites are licensed by distributive readings on definite plural DPs, for instance, they are not licensed by distributive readings of indefinite bare plurals: A gyerekek hoztak egyegy könyvet 'The children brought a book each' vs. *Gyerekek játszottak egy-egy labdával 'Children were playing with a-a ball'.

7. We consider here only the version of some that occurs with count nouns and thus ignore 'mass' some exemplified in Martin drank some wine. The modifier use of some exemplified in E.B. White's Some pig! will also be ignored.

8. The intermediate reading of this sentence is problematic for the claim in Schwartzchild 2002, according to which indefinites scoping out of an island are to be analyzed as singleton indefinites.

9. The derogatory flavor and the possibility of being modified by or other are properties that differentiate some from all the other indefinite Ds in English.

10. A variable bound by a quantifier or in the immediate scope of negation cannot be iden- 
tified because in order for it to be identified, all the possibilities in the context should have the same singleton value set for the variable in question. This situation is incompatible with the a variable having to cover its value set.

11. I owe this observation to Polly Jacobson.

12. The notion of 'non-trivial property', taken from de Hoop's 1995 insightful discussion of enkele and sommige in Dutch is meant to rule out the trivial property of being identical with oneself and any other properties that all entities have in all worlds.

13. Taking $\mathrm{E}_{j, w}$ as the domain over which the implicit universal involved in want quantifies is an oversimplification. Just like in the case of counterfactual conditionals, the domain has to go beyond this set in certain cases. Thus, even if it is known by both you and me that I will buy a particular house, 130 on Sherman St. in Santa Cruz and no other, if I say I want to buy a house in Santa Cruz because I like to live there we may interpret the variable introduced by a house as having narrowest scope and therefore given multiple values. What is involved is a limited expanding of the epistemic base of the subject by disregarding certain facts.

14. 'Strong' existential assertions such as [75a] differ from 'weak' ones such as those mentioned in the outset in [1] in that the former but not the latter are incompatible with marked indefinites. Thus, [75b] contrasts with Mary has some cousin who is Harrison Ford's sisterin-law. (For reasons that are unclear to me, using brother or sister instead of cousin here is much less appropriate.) Strong existential assertions, I assume, affirm existence and involve a property-denoting pivot. Weak existentials introduce a discourse referent contributed by their pivot DP. Note also that the use of $a(n)$ in these examples is different from the use of the definite determiner with proper names in languages that permit or require it. Proper names introduce a variable. The question in their case is whether they may do it on their own or need a D to do so.

\section{References}

Abusch, D. and M. Rooth, 1997, Epistemic NP modifiers, in SALT 7; 1997, pp. 1 $-27$.

Dekker, P., 1993, Transsentential Meditations, University of Amsterdam dissertation.

Farkas, D., 1997, Dependent indefinites, in F. Corblin, D. Godard, and J.M. Marandin (eds.), Empirical Issues in Formal Syntax and Semantics, Peter Lang Publishers, pp. 243-268.

Farkas, D., (to appear), Extreme non-specificity in Romanian, to appear in Drijkoningen, F. (ed.), Proceedings from Going Romance, Utrecht, December 2001.

Geurts, B., 2000, Indefinites and Choice Functions, in Linguistic Inquiry 4, pp. 731-739. Giannakidou, A., 1998, Polarity Sensitivity as (Non)Veridical Dependency, John Benjamins Publishing.

Giannakidou, A., 2001, The meaning of free choice, in Linguistics and Philosophy 24, pp. 659-735.

Hawkins, J., 1991, On (in)definite articles: implicatures and (un)grammaticality prediction, in Journal of Linguistics 27, pp. 405 - 442.

Groenendijk, J., M. Stokhof, and F. Veltman, 1996, Coreference and Modality, in S. 
Lappin (ed.), The Handbook of Contemporary Semantic Theory, Blackwell, pp. 179-215.

Gunlogson, C., 2001, True to Form, PhD dissertation, UCSC.

Heim, I., 1982, The Semantics of Definite and Indefinite Noun Phrases, University of Massachusetts dissertation.

Heim, I., 1992, Presupposition projection and the semantics of attitude verbs, in Journal of Semantics 9, pp. 183-221.

Hintikka, J. 1986, The semantics of a certain, in Linguistic Inquiry 17, pp. 331336.

de Hoop, H. 1995, On the characterization of the weak-strong distinction, in Bach, E., E. Jelinek, A. Kratzer, B. Partee (eds.), Quantification in Natural Languages, Kluwer, Dordrecht, pp. 421-450.

Horn, L. R., 1999, any and (-)ever: Free choice and free relatives, ms, June 17, 1999.

Israel, M., ms., Some and the pragmatics of indefinite construal.

Jayez, J. and L. Tovena (this volume), Determiners and (Un)certainty.

Kadmon, N. and F. Landman, 1993, Any, in Linguistics and Philosophy 16, pp. 353-422.

Kamp, H. and A. Bende-Farkas, Indefinites and Binding: from Specificity to Incorporation, Lecture notes, ESSLLI01.

Kratzer, A., 1998, Scope or Pseudo-Scope? Are there Wide-Scope Indefinites?, in S. Rothstein (ed.) Events in Grammar, Kluwer, Dordrecht, pp. 163-196.

Ladusaw, W., 1980, Polarity Sensitivity as Inherent Scope Relations, Garland, New York.

Langacker, R., 1991, Foundations of Cognitive Grammar: volume II, descriptive application. Stanford University Press.

McCawley, J. D., 1981, —em Everything That Linguists Have Always Wanted to Know about Logic - But Were Ashamed to Ask, University of Chicago Press.

Prince, E., 1981, On the inferencing of indefinite-this NPs. In Elements of discourse understanding, A. K. Joshi et al. (eds.), Cambridge University Press, pp. 231 - 250.

Reinhart, T., 1997, Quantifier Scope: How Labor is Divided Between QR and Choice-Functions, in Linguistics and Philosophy 20, pp. 335-397.

Schwarzschild, R., 2001, Singleton indefinites, to appear in Journal of Semantics.

Szabolcsi, A., Positive Polarity - Negative Polarity, ms., 2001. 